\title{
1 Deep learning predicts post-surgical recurrence of hepatocellular carcinoma from digital
}

\section{2 whole-slide images}

3 Rikiya Yamashita ${ }^{1,2}$, Jin Long ${ }^{2}$, Atif Saleem ${ }^{3}$, Daniel L. Rubin ${ }^{1,2 \S}$, Jeanne Shen ${ }^{2,3 \S^{*}}$

$4{ }^{1}$ Department of Biomedical Data Science, Stanford University School of Medicine, 1265 Welch

5 Road, Stanford, CA 94305, USA

$6 \quad{ }^{2}$ Center for Artificial Intelligence in Medicine and Imaging, Stanford University, 1701 Page Mill

7 Road, Palo Alto, CA 94304, USA

$8{ }^{3}$ Department of Pathology, Stanford University School of Medicine, 300 Pasteur Drive,

9 Stanford, CA 94305, USA

$10 \S$ Equal contribution

$11 *$ Address correspondence to:

16 Word count: 3,025 
medRxiv preprint doi: https://doi.org/10.1101/2020.08.22.20179952; this version posted August 25, 2020. The copyright holder for this preprint (which was not certified by peer review) is the author/funder, who has granted medRxiv a license to display the preprint in perpetuity.

It is made available under a CC-BY-NC-ND 4.0 International license .

\section{Abstract}

18 Recurrence risk stratification of patients undergoing primary surgical resection for hepatocellular

19 carcinoma (HCC) is an area of active investigation, and several staging systems have been

20 proposed to optimize treatment strategies. However, as many as $70 \%$ of patients still have tumor

21 recurrence at 5 years post-surgery. Routine hematoxylin and eosin (H\&E)-stained histopathology

22 slides may contain morphologic features associated with tumor recurrence. In this study, we

23 developed and independently validated a deep learning-based system (HCC-SurvNet) that

24 provides risk scores for disease recurrence after primary surgical resection, directly from H\&E-

25 stained digital whole-slide images of formalin-fixed, paraffin embedded liver resections. Our

26 model achieved a concordance index of 0.724 on a held-out internal test set of 53 patients, and

270.683 on an external test set of 198 patients, exceeding the performance of standard staging using

28 the American Joint Committee on Cancer (AJCC)/International Union against Cancer (UICC)

29 Tumor-Node-Metastasis (TNM) classification system, on both the internal and external test

30 cohorts ( $p=0.018$ and 0.025 , respectively). We observed statistically significant differences in the

31 survival distributions between low- and high-risk subgroups, as stratified by the risk scores

32 predicted by HCC-SurvNet on both the internal and external test sets (log-rank p-value: 0.0013

33 and $<0.0001$, respectively). On multivariable Cox proportional hazards analysis, the risk score

34 was an independent risk factor for post-surgical recurrence, on both the internal (hazard ratio

$35(\mathrm{HR})=7.44(95 \% \mathrm{CI}: 1.60,34.6), \mathrm{p}=0.0105)$ and external $(\mathrm{HR}=2.37(95 \% \mathrm{CI}: 1.27,4.43)$,

$36 \mathrm{p}=0.0069)$ test sets. Our results suggest that deep learning-based models can provide recurrence

37 risk scores which may augment current patient stratification methods, and help refine the clinical

38 management of patients undergoing primary surgical resection for HCC. 
medRxiv preprint doi: https://doi.org/10.1101/2020.08.22.20179952; this version posted August 25, 2020. The copyright holder for this preprint (which was not certified by peer review) is the author/funder, who has granted medRxiv a license to display the preprint in perpetuity.

It is made available under a CC-BY-NC-ND 4.0 International license .

Introduction

41 Hepatocellular carcinoma (HCC) is the most prevalent primary liver malignancy and the fourth

42 leading cause of cancer-related death worldwide. ${ }^{1,2}$ Despite advances in prevention, surveillance,

43 early detection, and treatment, its incidence and cancer-specific mortality continue to rise, with

44 the majority of patients still presenting at advanced stages. ${ }^{1,2}$ To stratify patients according to

45 their expected outcome in order to optimize treatment strategies, several staging systems, such as

46 the American Joint Committee on Cancer (AJCC)/International Union against Cancer (UICC)

47 Tumor-Node-Metastasis (TNM) ${ }^{3}$ and the Barcelona Clinic Liver Cancer (BCLC) system, ${ }^{4}$ have

48 been proposed and validated. However, as many as $70 \%$ of patients still have tumor recurrence

49 within 5 years post-treatment, ${ }^{2,5-7}$ including both true recurrence due to intrahepatic metastasis

50 and de novo primary cancers arising in the background liver, as the majority of HCCs occur in

51 patients with underlying chronic liver disease that directly contributes to the development of

52 HCC. Therefore, further refinement and improvement of recurrence risk stratification is

53 warranted.

54 Histopathologic assessment plays a key role in recurrence risk stratification, as it evaluates

55 human-recognizable morphologic features associated with tumor recurrence, such as

56 histopathologic grade and vascular invasion. ${ }^{8-11}$ Prognostic nomograms for prediction of

57 recurrence after curative liver resection for $\mathrm{HCC}$ have been proposed using clinicopathologic

58 variables. ${ }^{12}$ However, histopathologic features are interpreted by pathologists, which is subject to

59 reproducibility problems (an example being inter- and intra-observer variability in the

60 assessment of microvascular invasion $\left.{ }^{13}\right)$. On the other hand, recent advances in computer vision,

61 deep learning, and other forms of machine learning have enabled the identification of

62 histomorphologic patterns and features informative of disease outcomes which are not readily 
63 recognizable by the human eye, and which are reproducible. Thus, there has been much interest

64 in applying computer vision methods to histologic images for automated outcome prediction. ${ }^{14-21}$

65 Mobadersany et al. ${ }^{14}$ and Zhu et al. ${ }^{15}$ applied convolutional neural networks, a type of deep

66 learning network, to predict patient survival directly from histopathologic images of brain and

67 lung cancers, respectively. In these two studies, to achieve direct survival prediction from

68 histopathologic images, the negative partial log-likelihood was used as the loss function, which

69 enabled the models to output the risk values of the Cox proportional hazard model's exponential

70 part. Saillard et al. ${ }^{21}$ recently developed a deep learning-based model for the prediction of overall

71 survival after surgical resection in patients with $\mathrm{HCC}$, using digital whole-slide images.

72 However, no studies to date have sought to predict post-surgical recurrence of HCC directly

73 from histopathologic images using deep learning.

74 In this study, we developed and independently validated a deep convolutional neural network for

75 predicting risk scores for the recurrence-free interval (RFI) after curative-intent surgical

76 resection for $\mathrm{HCC}$, directly from digital whole-slide images (WSI) of hematoxylin and eosin

77 (H\&E)-stained, formalin-fixed, paraffin embedded (FFPE) primary liver resections. We built on

78 and extended the aforementioned prior work by applying the negative partial log-likelihood as a

79 loss function, so that the model outputs risk scores for post-surgical recurrence. In doing so, we

80 present a fully automated approach to $\mathrm{HCC}$ recurrence risk prognostication on histopathologic

81 images, which can be adopted for use in clinical settings to refine treatment and follow-up plans.

\section{Results}

83 An overall framework for the deep learning-based system for predicting the risk score for RFI,

84 hereafter referred to as HCC-SurvNet, is shown in Figure 1. The system consists of two stages,

85 i.e. tumor tile classification and risk score prediction. 
medRxiv preprint doi: https://doi.org/10.1101/2020.08.22.20179952; this version posted August 25, 2020. The copyright holder for this preprint (which was not certified by peer review) is the author/funder, who has granted medRxiv a license to display the preprint in perpetuity.

It is made available under a CC-BY-NC-ND 4.0 International license .

Tumor tile classification

87 To develop a deep convolutional neural network $(\mathrm{CNN})$ to automatically detect tumor-containing

88 tiles within WSI, we used the Stanford-HCCDET ( $\mathrm{n}=128,222$ tiles from 36 WSI) dataset. All

89 tumor regions in each WSI in the Stanford-HCCDET dataset were manually annotated by the

90 reference pathologist (J.S.). Each WSI was preprocessed and tiled into image patches. Using

91 these ground truth labels and image tiles, we trained and tested a CNN using 78\% of WSI in the

92 Stanford-HCCDET for training, 11\% for validation, and 11\% for internal testing, with no patient

93 overlap between any of these three sets. The final optimized tumor versus non-tumor tile

94 classifier was externally tested on 30 WSI ( $n=82,532$ tiles) randomly sampled from the TCGA-

95 HCC dataset.

96 Among the tiles in the internal test set, $25.7 \%$ (2,932 of 11,412 tiles) were tumor positive,

97 whereas $48.8 \%(40,288$ out of 82,532 tiles $)$ were tumor positive in the external test set. The

98 accuracies of tumor tile classification were $92.3 \%$ and $90.8 \%$ for the internal and external test

99 sets, respectively. The areas under the receiver-operating-characteristic-curve (AUROCs) were

$100 \quad 0.952(95 \%$ CI: $0.948,0.957)$ and $0.956(95 \%$ CI: $0.955,0.958)$ for the internal and external test

101 sets, respectively. Model outputs showed a statistically significant difference between tiles with a

102 ground truth of tumor versus non-tumor, on both the internal and external test sets $(\mathrm{p}<0.0001$ and

$103 \mathrm{p}<0.0001$, respectively) (Figures 2 and 3).

104 Risk score prediction

\section{$\underline{\text { Datasets }}$}

106 To develop a risk score prediction model, we used two datasets: the TCGA-HCC and Stanford-

107 HCC datasets, originating from two independent data sources, the Cancer Genome Atlas 
medRxiv preprint doi: https://doi.org/10.1101/2020.08.22.20179952; this version posted August 25, 2020. The copyright holder for this preprint (which was not certified by peer review) is the author/funder, who has granted medRxiv a license to display the preprint in perpetuity.

It is made available under a CC-BY-NC-ND 4.0 International license .

108 (TCGA)-LIHC diagnostic slide collection and the Stanford Department of Pathology slide 109 archive, respectively. The TCGA-HCC was further split into TCGA-HCC development and test 110 datasets.

111 The TCGA-HCC development dataset (containing the training and validation sets) consisted of 112299 patients (median age of 60 years, with an interquartile range (IQR) of 51-68 years, 69\% male 113 and $31 \%$ female). The frequencies of risk factors for $\mathrm{HCC}$ were: $32 \%$ for hepatitis B virus 114 infection, $15 \%$ for hepatitis C virus infection, 34\% for alcohol intake, and $4.9 \%$ for NAFLD. The 115 AJCC ( $8^{\text {th }}$ edition) stage grouping was IA in $2.7 \%$, IB in $41 \%$, II in $29 \%$, IIIA in $20 \%$, IIIB in $1165.4 \%$, IVA in $1.0 \%$, and IVB in $0.3 \%$ of the patients, respectively. One hundred and fifty-one 117 patients experienced disease recurrence during follow-up (median follow-up time of 12.2 118 months) (Table 1).

119 The TCGA-HCC test dataset consisted of 53 patients (median age of 61 years, with an IQR of 120 51-68 years, $62 \%$ male and 38\% female). The frequencies of risk factors for HCC were: $33 \%$ for 121 hepatitis B virus infection, $16 \%$ for hepatitis $\mathrm{C}$ virus infection, $39 \%$ for alcohol intake, and $10 \%$ 122 for NAFLD. The AJCC stage grouping was IA in $1.9 \%$, IB in $46 \%$, II in $31 \%$, IIIA in $17 \%$, IIIB 123 in $1.9 \%$, and IVB in $1.9 \%$ of the patients. Twenty-five patients experienced recurrence during 124 follow-up (median follow-up time of 12.7 months) (Table 1). None of the clinicopathologic 125 features were significantly associated with shorter RFI upon univariable Cox regression analysis, 126 while a Batts-Ludwig ${ }^{22}$ fibrosis stage $>2$ showed borderline significance (hazard ratio $(\mathrm{HR})=2.7$ 127 (95\% confidence interval $(\mathrm{CI}) 0.98,7.7), \mathrm{p}=0.0543)$ (Table 2$)$.

128 The Stanford-HCC dataset consisted of 198 patients (median age of 64 years with an IQR of 5712969 years, $79 \%$ male and $21 \%$ female). The frequencies of risk factors for HCC were: $26 \%$ for 
medRxiv preprint doi: https://doi.org/10.1101/2020.08.22.20179952; this version posted August 25, 2020. The copyright holder for this preprint (which was not certified by peer review) is the author/funder, who has granted medRxiv a license to display the preprint in perpetuity.

It is made available under a CC-BY-NC-ND 4.0 International license .

130 hepatitis $\mathrm{B}$ virus infection, $52 \%$ for hepatitis $\mathrm{C}$ virus infection, $8.6 \%$ for alcohol intake, and $7.1 \%$

131 for NAFLD. The overall AJCC stage grouping was IA in $22 \%$, IB in $21 \%$, II in $34 \%$, IIIA in

$1325.6 \%$, IIIB in $3.5 \%$, and IVA in $1.0 \%$ of the patients, respectively. Sixty-two patients

133 experienced disease recurrence during follow-up (median follow-up time of 24.9 months) (Table

134 1). The clinical and pathologic features associated with shorter RFI were AJCC stage grouping $>$

135 II $(\mathrm{HR}=4.4(95 \% \mathrm{CI} 2.3,8.3), \mathrm{p}<0.0001)$, greatest tumor diameter $>5 \mathrm{~cm}(\mathrm{HR}=3.5(95 \%$ CI 2.1 ,

136 5.8), $\mathrm{p}<0.0001)$, histologic grade $>$ moderately differentiated $(\mathrm{HR}=2.1(95 \% \mathrm{CI} 1.2,3.9)$,

$137 \mathrm{p}=0.0128)$, presence of microvascular invasion $(\mathrm{HR}=3.9(95 \% \mathrm{CI} 2.4,6.5), \mathrm{p}<0.0001)$, presence

138 of macrovascular invasion $(\mathrm{HR}=5.3(95 \% \mathrm{CI} 2.1,13), \mathrm{p}<0.0001)$, positive surgical margin

$139(\mathrm{HR}=6.8(95 \% \mathrm{CI} 1.6,28), \mathrm{p}=0.009)$, and fibrosis stage $>2(\mathrm{HR}=0.33(95 \% \mathrm{CI} 0.2,0.55)$,

$140 \mathrm{p}<0.0001)$ using univariable Cox regression analysis (Table 2).

HCC-SurvNet performance for RFI prediction

142 The tumor tile classification model was applied to each tissue-containing image tile in the

143 TCGA-HCC development ( $\mathrm{n}=299$ WSIs) and test ( $\mathrm{n}=53$ WSIs) datasets and the Stanford-HCC

144 dataset ( $\mathrm{n}=198$ WSIs). From each WSI, the 100 tiles with the highest probabilities for the tumor

145 class were selected for input into the subsequent risk score model. Figure 4 shows examples of

146 tiles with probabilities in the top 100 for containing tumor, overlaid onto the original WSI. A

147 MobileNetV2 ${ }^{23}$ pre-trained on ImageNet ${ }^{24}$ was modified by replacing the fully-connected layers,

148 and fine-tuned by transfer learning with on-the-fly data augmentation on the tiles from the

149 TCGA-HCC development dataset (n=307 WSI from 299 patients), where the model input was a

$150299 \times 299$ pixel image tile, and the output was a continuous tile-level risk score from the hazard

151 function for RFI. The negative partial log-likelihood of the Cox proportional hazards model was

152 used as a loss function. ${ }^{14,15}$ The model's performance was evaluated internally on the TCGA- 
medRxiv preprint doi: https://doi.org/10.1101/2020.08.22.20179952; this version posted August 25, 2020. The copyright holder for this preprint (which was not certified by peer review) is the author/funder, who has granted medRxiv a license to display the preprint in perpetuity.

It is made available under a CC-BY-NC-ND 4.0 International license.

153 HCC test dataset ( $\mathrm{n}=53 \mathrm{WSI}$ from 53 patients), and externally on the Stanford-HCC dataset

154 ( $\mathrm{n}=198$ WSI from 198 patients). All tile-level risk scores from a patient were averaged to yield a

155 patient-level risk score.

156 We assessed HCC-SurvNet's performance using Harrell's ${ }^{25}$ and Uno's ${ }^{26}$ concordance indices (c-

157 indices). On the internal test set (TCGA-HCC test dataset, $\mathrm{n}=53$ patients), Harrell's and Uno's c-

158 indices were 0.724 and 0.724 , respectively. On the external test set (Stanford-HCC, $\mathrm{n}=198$

159 patients), the indices were 0.683 and 0.670 , respectively. We observed statistically significant

160 differences in the survival distributions between the low- and high-risk subgroups, as stratified

161 by the risk scores predicted by HCC-SurvNet, on both the internal and external test sets (log-rank

162 p-value: 0.0013 and $<0.0001$, respectively) (Figures 5, 6). Histograms of HCC-SurvNet's risk

163 scores, along with the threshold used for risk group stratification, are shown in Supplementary

164 Figure 1. On univariable Cox proportional hazards analysis, the HCC-SurvNet risk score was a

165 predictor of the RFI, for both the internal $(\mathrm{HR}=6.52(95 \% \mathrm{CI}: 1.83,23.2), \mathrm{p}=0.0038)$ and

166 external $(\mathrm{HR}=3.72(95 \% \mathrm{CI}: 2.17,6.37), \mathrm{p}<0.0001)$ test sets (Table 2$)$. A continuous linear

167 association between HCC-SurvNet's risk score and the log relative hazard for RFI was observed

168 by analysis of the internal and external test cohorts by univariable Cox proportional hazards

169 regression with restricted cubic splines (Supplementary Figure 2), validating the use of HCC-

170 SurvNet's risk score as a linear factor in the Cox analyses.

171 On multivariable Cox proportional hazards analysis, HCC-SurvNet's risk score was an

172 independent predictor of the RFI, for both the internal (HR=7.44 (95\% CI: 1.60, 34.6),

$173 \mathrm{p}=0.0105)$ and external $(\mathrm{HR}=2.37(95 \% \mathrm{CI}: 1.27,4.43), \mathrm{p}=0.00685)$ test sets (Table 3$)$. No other

174 clinicopathologic variable was statistically significant on the internal test set. Microvascular

175 invasion $(\mathrm{HR}=2.84(95 \% \mathrm{CI}: 1.61,5.00), \mathrm{p}=0.000294)$ and fibrosis stage $(\mathrm{HR}=0.501(95 \% \mathrm{CI}$ : 
medRxiv preprint doi: https://doi.org/10.1101/2020.08.22.20179952; this version posted August 25, 2020. The copyright holder for this preprint (which was not certified by peer review) is the author/funder, who has granted medRxiv a license to display the preprint in perpetuity.

It is made available under a CC-BY-NC-ND 4.0 International license .

$0.278,0.904), p=0.0217$ ) showed statistical significance on the external test set, along with HCC-

177 SurvNet's risk score. Schoenfeld's global test showed p-values greater than 0.05 on both the

178 internal $(p=0.083)$ and external $(p=0.0702)$ test sets. On mixed-effect Cox regression analysis

179 with the TCGA institution as a random effect, HCC-SurvNet's risk score was an independent

180 predictor $(\mathrm{p}=0.014)$, along with the histologic grade $(\mathrm{p}=0.014)$ and macrovascular invasion

181 ( $\mathrm{p}=0.013)$. In the external test (Stanford-HCC) cohort, HCC-SurvNet's risk score was positively

182 associated with the AJCC stage grouping, greatest tumor diameter, and microvascular invasion,

183 and negatively associated with fibrosis stage (Table 4). HCC-SurvNet's risk score yielded a

184 significantly higher Harrell's c-index ( 0.72 for the internal and 0.68 for the external test cohort)

185 than that obtained using the AJCC Stage grouping ( 0.56 for the internal and 0.60 for the external

186 test cohort), on both the internal and external test cohorts ( $\mathrm{p}=0.018$ and 0.025 , respectively).

187 Discussion

188 Building upon recent advances in deep learning, we have developed a system for predicting RFI

189 after curative-intent surgical resection in patients with HCC, directly from H\&E-stained FFPE

190 WSI. The system outputs an RFI risk score by first applying a deep CNN to automatically detect

191 tumor-containing tiles. Then, a second model outputs a continuous risk score based on analysis

192 of the top 100 tumor-containing tiles from each WSI. In the internal and external test cohorts, we

193 observed statistically significant differences in the survival distributions between the low- and

194 high-risk subgroups, as stratified by the risk score predicted by the system. The results of

195 multivariable analyses indicate that the HCC-SurvNet risk score could help supplement

196 established clinicopathologic predictors of RFI, thereby improving recurrence risk stratification. 
medRxiv preprint doi: https://doi.org/10.1101/2020.08.22.20179952; this version posted August 25, 2020. The copyright holder for this preprint (which was not certified by peer review) is the author/funder, who has granted medRxiv a license to display the preprint in perpetuity.

It is made available under a CC-BY-NC-ND 4.0 International license .

197 In the present study, HCC-SurvNet significantly outperformed the standard AJCC/UICC staging

198 system in predicting the post-surgical HCC recurrence risk. Shim et al. ${ }^{12}$ reported the

199 performance of a prognostic nomogram for recurrence prediction after curative liver resection in

200 HCC patients, which yielded a c-index of 0.66 for 2-year recurrence on an independent

201 validation cohort. Although a direct comparison with the performance of their nomogram is not

202 possible, as their patient cohort was different from ours, our risk score appears to have a

203 performance that is on par with, or slightly better than, the prognostic nomogram.

204 Advances in deep learning, and other forms of machine learning, have led to the identification of

205 histomorphologic features informative of disease outcomes, and prior works have applied these

206 methods to automated outcome prediction. ${ }^{14-21}$ The automatic extraction of such features directly

207 from WSI has the potential to add value to current treatment planning paradigms by increasing

208 both the accuracy of prognostic risk stratification and the objectivity and reproducibility of

209 biomarker assessment. Mobadersany et al. ${ }^{14}$ and Zhu et al. ${ }^{15}$ previously applied convolutional

210 neural networks to survival prediction directly from histopathologic images, by integrating the

211 negative partial log-likelihood into the model as a loss function, which enables the model to

212 output a value that can be regarded as a prognostic risk score. However, in these prior studies,

213 representative tiles were manually identified for input into the deep learning models. This

214 requirement for manual tile selection, even during inference, makes such models less practical

215 for widespread clinical deployment. In this work, we present a system which automatically

216 selects representative image tiles, which should increase the ease of deployment in clinical

217 settings. 
medRxiv preprint doi: https://doi.org/10.1101/2020.08.22.20179952; this version posted August 25, 2020. The copyright holder for this preprint (which was not certified by peer review) is the author/funder, who has granted medRxiv a license to display the preprint in perpetuity.

It is made available under a CC-BY-NC-ND 4.0 International license .

218 Saillard et al. ${ }^{21}$ were the first to apply deep learning to digital H\&E WSI to predict overall

219 survival after resection in HCC patients. On their external test set (342 WSI from the TCGA),

220 their models yielded c-indices of 0.68 and 0.70 for overall survival prediction. We were unable

221 to perform a direct comparison with their models, as the outcomes and datasets used were

222 different, but our c-index on the external test set of 0.68 appears comparable to that reported for

223 their model. In their study, they applied a CNN pre-trained on ImageNet as a fixed feature

224 extractor. The features extracted were optimized for natural, rather than histopathologic, images,

225 suggesting that there might be further potential for improving prediction performance by

226 optimizing feature extraction for histopathologic images. ${ }^{27}$ To leverage the full capacity of our

227 HCC-SurvNet deep learning system, we fine-tuned all of the models' parameters, including those

228 for feature extraction (i.e. the convolutional blocks), with histopathologic images. Whereas

229 models to date have focused on predicting overall survival, ours focused on the recurrence-free

230 interval, as the intent was to aid refinement of treatment strategies by providing a risk score that

231 was specific for HCC recurrence and/or HCC-related mortality after curative-intent surgical

232 resection.

233 A specific strength of our study was the review and confirmation of all clinicopathologic

234 variables in the TCGA-HCC cohort and re-coding of older edition AJCC classifications to the

235 latest $8^{\text {th }}$ edition classification. Previous other studies ${ }^{19}$ have also developed models for the

236 prediction of overall survival in post-surgical HCC patients, also by using clinicopathologic data

237 from the TCGA. However, use of TCGA clinicopathologic data presents some significant

238 limitations, which are often overlooked. These include the fact that the AJCC TNM

239 classifications used across cases in the TCGA-LIHC dataset range from the $4^{\text {th }}$ through the $7^{\text {th }}$

240 editions, resulting in inconsistency in the meaning of the pathologic $\mathrm{T}, \mathrm{N}$, and $\mathrm{M}$ categories 
medRxiv preprint doi: https://doi.org/10.1101/2020.08.22.20179952; this version posted August 25, 2020. The copyright holder for this preprint (which was not certified by peer review) is the author/funder, who has granted medRxiv a license to display the preprint in perpetuity.

It is made available under a CC-BY-NC-ND 4.0 International license .

241 across different patients resected during different time periods. In addition, the pathology reports

242 in TCGA-LIHC came from different institutions with wide variation in the reporting of

243 pathologic features. Therefore, prior to use of TCGA data, standardization, in particular, of all

244 pathologic variables, as performed in this study, is necessary. As the TCGA data were collected

245 from 35 different institutions, each with different H\&E staining and digitization protocols, we

246 constructed a mixed-effect Cox model to account for potential intraclass correlations present

247 between WSI originating from the same institution. After taking the originating institution into

248 account as a random effect, we found that HCC-SurvNet's risk score remained an independent

249 predictor of recurrence-free interval, along with the histologic grade and the presence of

250 macrovascular invasion.

251 A limitation of our study was that the dataset used to externally evaluate HCC-SurvNet's

252 performance was restricted to cases from a single institution. Due to limitations in the datasets

253 that were available to us, we chose to reserve the more heterogeneous, multi-institutional TCGA-

254 HCC dataset for HCC-SurvNet model development, with the intention of capturing

255 histomorphologic features informative of HCC recurrence which were robust to inter-

256 institutional variations in H\&E staining and scanning protocols. With further development and

257 validation on larger, more diverse datasets, we hope that risk scores produced by HCC-SurvNet,

258 as well as other similar deep learning-based models, might one day offer clinical value as a

259 supplement to currently-established clinicopathologic predictors of recurrence and survival.

260 Another limitation was the black-box nature of deep learning systems. To gain insights into

261 model interpretability, we assessed the associations between HCC-SurvNet's risk score and

262 different patient characteristics in the external test (Stanford-HCC) cohort. The HCC-SurvNet

263 risk score was significantly associated with several well-recognized prognostic factors, including 
medRxiv preprint doi: https://doi.org/10.1101/2020.08.22.20179952; this version posted August 25, 2020. The copyright holder for this preprint (which was not certified by peer review) is the author/funder, who has granted medRxiv a license to display the preprint in perpetuity.

It is made available under a CC-BY-NC-ND 4.0 International license .

264 the AJCC stage grouping, largest tumor diameter, microvascular invasion, and Batts-Ludwig

265 fibrosis stage. In addition, the independent contribution of the HCC-SurvNet risk score to

266 recurrence-free interval prediction, when analyzed together with other known clinicopathologic

267 variables in the multivariable Cox regression, suggests that HCC-SurvNet was able to extract

268 some as-yet unrecognized histomorphologic features informative of recurrence, which might

269 have biological significance and correlate with other important outcomes, such as response to

270 adjuvant treatment. It remains for future studies to explore the additional potential of deep

271 learning for prognostication and treatment response prediction in $\mathrm{HCC}$, and other malignancies.

272 In conclusion, we have shown that a deep learning-based cancer recurrence risk score extracted

273 from routine H\&E WSI of primary surgical resections for HCC independently predicts the RFI,

274 and significantly outperforms the most commonly-used standard AJCC/UICC stage grouping.

275 With further validation on larger, more diverse datasets, such a risk score could augment current

276 methods for predicting the risk of HCC recurrence after primary surgical resection, thereby

277 assisting clinicians in tailoring post-surgical management.

\section{Methods}

279 Patient population

280 A total of 250 primary hepatic resection specimens ( $\mathrm{n}=250$ patients) from surgeries performed at

281 our institution between January 1, 2009 and December 31, 2017, with glass slides available for

282 retrieval from the departmental slide archive, were included in the dataset. Prior to digitization,

283 the time to recurrence after surgical resection, as well as patient demographic information (age at

284 surgical resection, gender, and alcohol intake), and clinicopathologic variables (history of

285 hepatitis B and C viral infection, non-alcoholic fatty liver disease (NAFLD), HCC multi- 
medRxiv preprint doi: https://doi.org/10.1101/2020.08.22.20179952; this version posted August 25, 2020. The copyright holder for this preprint (which was not certified by peer review) is the author/funder, who has granted medRxiv a license to display the preprint in perpetuity.

It is made available under a CC-BY-NC-ND 4.0 International license .

nodularity, macro- and micro- vascular invasion, largest tumor diameter, histologic World Health

291 because of lack of follow-up data after surgical resection, and three were excluded due to the

292 presence of comorbidities known to have contributed to the patients' deaths. This process

293 narrowed the final number of study patients down to 198. From each of these 198 patients, a

294 representative tumor H\&E slide (the one containing the highest grade of tumor in the specimen)

295 was digitized at high resolution (40x objective magnification, 0.25 micrometers per pixel) on an

296 Aperio AT2 scanner (Leica Biosystems, Nussloch, Germany), to generate a WSI in the SVS file

297 format. This dataset ( $\mathrm{n}=198 \mathrm{WSI}$, from 198 unique patients), referred to as the Stanford-HCC

298 dataset, was used for external evaluation of the risk score prediction model. From the excluded

299 patient pool (not included in Stanford-HCC), 36 patients were randomly selected, and a

300 representative tumor $H \& E$ slide from each patient was digitized using the exact same method as

301 described above, yielding a dataset with 36 WSI from 36 patients, referred to as the Stanford-

302 HCCDET dataset. This dataset was used to develop a model for automatically detecting tumor-

303 containing tiles in a WSI ("DET" stands for "detection"). Use of all patient material and data was

304 approved by the Stanford University Institutional Review Board, with waived informed consent.

305 In addition to the Stanford-HCC and Stanford-HCCDET datasets, a publicly-available dataset of

306379 FFPE diagnostic WSI from 365 unique patients in the TCGA-LIHC diagnostic slide

307 collection were downloaded via the GDC Data Portal ${ }^{29}$ and used to develop the risk score

308 prediction model for this study. The same patient demographics, clinicopathologic variables, and 
medRxiv preprint doi: https://doi.org/10.1101/2020.08.22.20179952; this version posted August 25, 2020. The copyright holder for this preprint (which was not certified by peer review) is the author/funder, who has granted medRxiv a license to display the preprint in perpetuity.

It is made available under a CC-BY-NC-ND 4.0 International license .

RFI as collected for Stanford-HCC were obtained through review of the accompanying metadata and pathology reports downloaded from the GDC Data Portal and the previously-published

311 Integrated TCGA Pan-Cancer Clinical Data Resource by Liu et al. ${ }^{30}$ RFI was defined as the

312 period from the date of surgery until the date of the first occurrence of a new tumor event, which

313 included progression of $\mathrm{HCC}$, locoregional recurrence, distant metastasis, new primary tumor, or

314 death with tumor. ${ }^{30}$ Patients who were alive without these events, or who died without tumor,

315 were censored. ${ }^{31}$ The event time was the shortest period from the date of surgery to the date of an

316 event. The censored time was the period from the date of surgery to the date of last contact with

317 the patient or the date of death without HCC. Given multiple changes to the AJCC classification

318 over the time period during which these specimens were collected (resulting in differences in the

319 pathologic staging criteria across different editions of the AJCC), a reference pathologist trained

320 in the interpretation of hepatobiliary pathology (J.S.) reviewed the WSI and the downloaded

321 pathology reports, in order to re-stage all of the patients based on the most current AJCC (8th

322 edition) classification. ${ }^{3}$ WSI scanned at 20x base magnification were excluded ( $\mathrm{n}=10 \mathrm{WSI}$, from

3234 patients). One patient ( $\mathrm{n}=1 \mathrm{WSI}$ ) with missing RFI was excluded. Seven patients ( $\mathrm{n}=7$ WSIs)

324 with mixed HCC-cholangiocarcinomas and one patient ( $\mathrm{n}=1 \mathrm{WSI}$ ) with an angiomyolipoma

325 were excluded from the dataset. The final dataset ( $\mathrm{n}=360 \mathrm{WSI}$, from 352 patients), referred to as

326 the TCGA-HCC dataset, contained patients from 35 institutions, each with potentially different

327 staining and scanning protocols. The TCGA-HCC dataset was randomly split into the

328 development cohort $(n=299$ patients: $n=247$ patients for training and $n=52$ patients for

329 validation) and internal test cohort ( $\mathrm{n}=53$ patients), with no patient overlap between the splits. 
medRxiv preprint doi: https://doi.org/10.1101/2020.08.22.20179952; this version posted August 25, 2020. The copyright holder for this preprint (which was not certified by peer review) is the author/funder, who has granted medRxiv a license to display the preprint in perpetuity.

It is made available under a CC-BY-NC-ND 4.0 International license .

331 First, tissue segmentation (i.e. tissue separation from white background) of the WSI was

332 performed by applying a combination of filters. Second, the WSI were tiled into image patches

333 with a size of $1024 \times 1024$ pixels, at a resolution of $40 \times(0.25 \mu \mathrm{m} /$ pixel $)$. Only the tiles

334 containing an overall tissue percentage of $>80 \%$ of the total surface area within each tile were

335 saved in PNG format. Lastly, the Vahadane method ${ }^{32}$ was used for stain normalization, to

336 convert all image tiles to a reference color space. All tiles were subsequently resized to $299 \mathrm{x}$

337299 pixels and used for the downstream analyses.

338 Tumor tile classification

339 All tumor regions in each WSI in the Stanford-HCCDET dataset were manually annotated by the

340 reference pathologist (J.S.) at 10x magnification, using Aperio ImageScope (Leica Biosystems,

341 Nussloch, Germany). Tiles containing both tumor and normal tissue were excluded from model

342 development and evaluation. Using these ground-truth annotated WSI, we developed a CNN for

343 automatically classifying an image tile into either the tumor or non-tumor class, where the model

344 input was a $299 \times 299$ pixel image tile in PNG format, and the output was a probability for each

345 class. The particular CNN architecture, PathCNN, which was originally proposed by Bilaloglu et

$346 \mathrm{al},{ }^{33}$ was trained and tested using the Stanford-HCCDET ( $\mathrm{n}=128,222$ tiles from 36 WSI) dataset,

347 with $78 \%$ of WSI used for training, 11\% used for validation, and $11 \%$ used as an internal test set,

348 with no patient overlap between any of these three sets). We used leaky ReLU ${ }^{34}$ with negative

349 slope 0.01 as the non-linearity. The dropout probability was set at 0.1 . The trainable parameters

350 were initialized using a Xavier weight initialization scheme, ${ }^{35}$ and updated using an Adam

351 optimization method ${ }^{36}$ with an initial learning rate of 0.001 . We applied stepwise learning rate

352 decay with a step size of 7 and gamma of 0.1 . The number of epochs was set at 25 , with a mini-

353 batch size of 32. A binary cross entropy loss function was applied. Input images were normalized 
medRxiv preprint doi: https://doi.org/10.1101/2020.08.22.20179952; this version posted August 25, 2020. The copyright holder for this preprint (which was not certified by peer review) is the author/funder, who has granted medRxiv a license to display the preprint in perpetuity.

It is made available under a CC-BY-NC-ND 4.0 International license .

354 by ((image -0.5$) / 0.5)$ before passing them to the model. We augmented the training data by

355 randomly introducing positional transforms: a horizontal flip and a rotation of $0^{\circ}, 90^{\circ}, 180^{\circ}$ or

$356270^{\circ}$ degrees. Additionally, we randomly adjusted the hue, brightness, contrast, and saturation of

357 the image. We used validation accuracy to select the final model. The final optimized tumor

358 versus non-tumor tile classifier was externally tested on 30 WSI ( $n=82,532$ tiles) randomly

359 sampled from the TCGA-HCC dataset. Of note, there was no patient overlap between the

360 Stanford-HCCDET and Stanford-HCC datasets, where the latter was used in the downstream

361 development of the risk score prediction model. The tumor tile classification model was

362 subsequently applied to each tissue-containing image tile in the Stanford-HCC ( $\mathrm{n}=198$ WSIs)

363 and TCGA-HCC ( $\mathrm{n}=360 \mathrm{WSIs})$ datasets. From each WSI, the 100 tiles with the highest

364 probabilities for the tumor class were selected for input into the subsequent survival analysis.

365 The value of 100 was chosen arbitrarily in order to incorporate enough representative tiles,

366 taking into account morphologic tumor heterogeneity in the WSI (Figure 4). Additional details

367 on the model's development are described in the Supplementary Methods.

368 HCC-SurvNet Development

369 The top 100 tiles selected by the tumor detector were used for the development of the risk score

370 model for RFI, which consisted of a MobileNetV2 ${ }^{23}$ pre-trained on ImageNet, ${ }^{24}$ modified by

371 replacing the fully-connected layers, and fine-tuned by transfer learning with on-the-fly data

372 augmentation on the tiles from the TCGA-HCC development dataset $(n=307$ WSI, $n=299$

373 patients), where the model input was a 299 x 299 pixel image tile in PNG format, and the output

374 was a continuous tile-level risk score from the hazard function for RFI. The dropout probability

375 in the replaced fully-connected classification layers was set at 0.7 . The trainable parameters were

376 fine-tuned using an AdamW optimization method ${ }^{37}$ with an initial learning rate of 0.001 . The 
medRxiv preprint doi: https://doi.org/10.1101/2020.08.22.20179952; this version posted August 25, 2020. The copyright holder for this preprint (which was not certified by peer review) is the author/funder, who has granted medRxiv a license to display the preprint in perpetuity.

It is made available under a CC-BY-NC-ND 4.0 International license .

377

378

379

380

381

382

383

384

385

386

387

\section{$\underline{\text { Statistical analysis }}$}

397 We summarized our study population with descriptive statistics, including the median and IQR

398 for continuous variables, and the proportion for categorical variables. The performance of the

number of epochs was set at 30 , with a mini-batch size of 80 . The negative partial log-likelihood of the Cox proportional hazards model was used as a loss function. ${ }^{14,15}$ Input images were normalized by ((image - mean) / standard deviation), where the mean and standard statistics were calculated for the ImageNet dataset before passing them to the model. We augmented the training data by randomly introducing positional transforms: a horizontal flip and a rotation of $0^{\circ}, 90^{\circ}, 180^{\circ}$ or $270^{\circ}$ degrees. Additionally, we randomly adjusted the hue, brightness, contrast, and saturation of the image. We used validation loss to select the final model. The model's performance was evaluated internally on the TCGA-HCC test dataset, and externally on the Stanford-HCC dataset. All tile-level risk scores from a patient were averaged to yield a patientlevel risk score. An overall framework for the system, referred to as HCC-SurvNet, is shown in Figure 1, with additional model development details described in the Supplementary Methods.

\section{$\underline{\text { Hardware and software }}$}

The PyTorch Python package (version 1.1.0) ${ }^{38}$ was used for model development. OpenSlide (version 3.4.1) ${ }^{39}$ was used to read WSI in the SVS format. Image preprocessing was performed on a High-Performance Computing (HPC) cluster operated by the Stanford Research Computing Center (Sherlock cluster: https://www.sherlock.stanford.edu/). Model development and evaluation were performed on a workstation with two GeForce RTX 2080 Ti (NVIDIA, Santa Clara, CA) graphics processing units, a Core i9-9820X (10 cores, $3.3 \mathrm{GHz}$ ) central processing unit (Intel, Santa Clara, CA), and 128 GB of random-access memory. 
medRxiv preprint doi: https://doi.org/10.1101/2020.08.22.20179952; this version posted August 25, 2020. The copyright holder for this preprint (which was not certified by peer review) is the author/funder, who has granted medRxiv a license to display the preprint in perpetuity.

It is made available under a CC-BY-NC-ND 4.0 International license .

tumor tile classification model was assessed using the overall accuracy and AUROC. Model outputs for tiles with a ground truth of tumor were compared with those for tiles with a ground

401 truth of non-tumor, using the Wilcoxon rank sum test. We evaluated the performance of the risk

402 score model using Harrell's ${ }^{25}$ and Uno's ${ }^{26}$ c-indices, which indicate better prediction when their

403 values approach one. Each patient was stratified into one of two subgroups (high-risk and low-

404 risk), based on their patient-level risk score. The median risk score on the validation set from

405 TCGA-HCC was used as the threshold for patient stratification (Supplementary Figure 1).

406 Kaplan-Meier analysis was performed, and a log-rank test was used to compare the survival

407 distributions between the subgroups. Univariable and multivariable Cox proportional hazards

408 models were used to assess the relationship between independent variables and RFI. The

409 independent variables included HCC-SurvNet's risk score, age at surgical resection, gender,

410 AJCC stage grouping, largest tumor diameter, tumor multifocality, histologic tumor grade,

411 microvascular invasion, macrovascular invasion, surgical margin status, fibrosis stage, and

412 history of Hepatitis B, Hepatitis C, alcohol intake, and non-alcoholic fatty liver disease. Of these,

413 variables with univariable p-values of less than 0.1 on either the internal or external test sets

414 were selected for inclusion in the multivariable analysis. The proportional hazards assumption

415 was checked using Schoenfeld's global test. To demonstrate the non-linear relationship between

416 HCC-SurvNet's risk score and the log relative hazard for RFI, univariable Cox proportional

417 hazards regression analysis with restricted cubic splines (3 knots) was performed. To account for

418 potential intraclass correlation among WSI prepared and scanned at the same institution within

419 the TCGA cohort, a mixed-effect Cox regression model was constructed using the institution as a

420 random effect. Spearman's correlation coefficients were computed to gain insight into

421 associations between the HCC-SurvNet risk score and different patient characteristics in the 
medRxiv preprint doi: https://doi.org/10.1101/2020.08.22.20179952; this version posted August 25, 2020. The copyright holder for this preprint (which was not certified by peer review) is the author/funder, who has granted medRxiv a license to display the preprint in perpetuity.

It is made available under a CC-BY-NC-ND 4.0 International license .

422 external test (Stanford-HCC) cohort. Harrell's c-index was compared between HCC-SurvNet's

423 risk score and the standard AJCC staging system, using a paired t-test.

424 A two-tailed alpha level of 0.05 was used for statistical significance. All statistical analyses were

425 performed using Python (v3.6.4, Python Software Foundation, https://www.python.org/) with the

426 lifelines (v0.24.0) and scikit-survival (v0.11) packages, as well as R (v3.6.3, R Foundation for

427 Statistical Computing, http://www.R-project.org/) with the survival (v3.1.12), coxme (v2.2.16),

428 pROC (v1.16.2), and rms (v5.1.4) packages.

429 Acknowledgments

430 This work was funded by the Stanford Departments of Pathology and Biomedical Data Science,

431 through a Stanford Clinical Data Science Fellowship to R.Y. Additional computational

432 infrastructure was provided by the Stanford Research Computing Center. We would also like to

433 thank Dr. Lu Tian, Stanford Department of Biomedical Data Science, for helpful initial

434 conversations regarding analysis planning.

\section{Author Contributions}

436 R.Y., D.L.R., and J.S. conceived and designed the study; R.Y. and J.S. performed the literature

437 search. R.Y., A.S. and J.S. performed the data collection; R.Y. performed the model

438 development and performance evaluation; R.Y. and J.L. performed the statistical analyses; R.Y.

439 drafted the manuscript; D.L.R. and J.S. supervised the study; all authors participated in the

440 critical revision and approval of the manuscript.

\section{Competing Interests}


medRxiv preprint doi: https://doi.org/10.1101/2020.08.22.20179952; this version posted August 25, 2020. The copyright holder for this preprint (which was not certified by peer review) is the author/funder, who has granted medRxiv a license to display the preprint in perpetuity.

It is made available under a CC-BY-NC-ND 4.0 International license .

442 The authors declare no competing interests.

\section{Data Availability}

444 All whole-slide-images for the TCGA cohort are publicly available at

445 https://portal.gdc.cancer.gov/. The Stanford whole-slide images are not publicly available, in

446 accordance with institutional requirements governing human subject privacy protection.

\section{Code availability}

448 All source code is available under an open-source license at:

449 https://github.com/RubinLab/HCCSurvNet

$451 \quad$ References

452 1. Yang, J. D. et al. A global view of hepatocellular carcinoma: trends, risk, prevention and

453 management. Nat. Rev. Gastroenterol. Hepatol. 16, 589-604 (2019).

$454 \quad 2 . \quad$ Forner, A., Reig, M. \& Bruix, J. Hepatocellular carcinoma. Lancet 391, 1301-1314

455 (2018).

456 3. Brierley, J., Gospodarowicz, M. K. (Mary K. . \& Wittekind, C. (Christian). TNM

457 Classification Of Malignant Tumours. 272 (Wiley-Blackwell, 2017).

458 4. Forner, A., Reig, M. E., de Lope, C. R. \& Bruix, J. Current strategy for staging and

459 treatment: the BCLC update and future prospects. Semin Liver Dis 30, 61-74 (2010).

460 5. Villanueva, A. Hepatocellular Carcinoma. N. Engl. J. Med. 380, 1450-1462 (2019). 
$461 \quad 6 . \quad$ Ishizawa, T. et al. Neither multiple tumors nor portal hypertension are surgical

462 contraindications for hepatocellular carcinoma. Gastroenterology 134, 1908-1916 (2008).

$4637 . \quad$ Hasegawa, K. et al. Comparison of resection and ablation for hepatocellular carcinoma: a

464 cohort study based on a Japanese nationwide survey. J. Hepatol. 58, 724-729 (2013).

$4658 . \quad$ Roayaie, S. et al. A system of classifying microvascular invasion to predict outcome after

466 resection in patients with hepatocellular carcinoma. Gastroenterology 137, 850-855 (2009).

467 9. Kamiyama, T. et al. Analysis of the risk factors for early death due to disease recurrence

468 or progression within 1 year after hepatectomy in patients with hepatocellular carcinoma. World

469 J Surg Oncol 10, 107 (2012).

470 10. Cucchetti, A. et al. Comparison of recurrence of hepatocellular carcinoma after resection

471 in patients with cirrhosis to its occurrence in a surveilled cirrhotic population. Ann. Surg. Oncol.

$47216,413-422(2009)$.

473 11. Colecchia, A. et al. Prognostic factors for hepatocellular carcinoma recurrence. World J.

474 Gastroenterol. 20, 5935-5950 (2014).

475 12. Shim, J. H. et al. Prognostic nomograms for prediction of recurrence and survival after

476 curative liver resection for hepatocellular carcinoma. Ann. Surg. 261, 939-946 (2015).

477 13. Rodríguez-Perálvarez, M. et al. A systematic review of microvascular invasion in 478 hepatocellular carcinoma: diagnostic and prognostic variability. Ann. Surg. Oncol. 20, 325-339 479 (2013). 
medRxiv preprint doi: https://doi.org/10.1101/2020.08.22.20179952; this version posted August 25, 2020. The copyright holder for this preprint (which was not certified by peer review) is the author/funder, who has granted medRxiv a license to display the preprint in perpetuity.

It is made available under a CC-BY-NC-ND 4.0 International license .

480 14. Mobadersany, P. et al. Predicting cancer outcomes from histology and genomics using

481 convolutional networks. Proc. Natl. Acad. Sci. USA 115, E2970-E2979 (2018).

482 15. Zhu, X., Yao, J. \& Huang, J. Deep convolutional neural network for survival analysis

483 with pathological images. in 2016 IEEE International Conference on Bioinformatics and

484 Biomedicine (BIBM) 544-547 (IEEE, 2016). doi:10.1109/BIBM.2016.7822579

485 16. Kather, J. N. et al. Predicting survival from colorectal cancer histology slides using deep

486 learning: A retrospective multicenter study. PLoS Med. 16, e1002730 (2019).

487 17. Kim, D. W. et al. Deep learning-based survival prediction of oral cancer patients. Sci.

488 Rep. 9, 6994 (2019).

489 18. Wulczyn, E. et al. Deep learning-based survival prediction for multiple cancer types

490 using histopathology images. PLoS One 15, e0233678 (2020).

491 19. Liao, H. et al. Classification and Prognosis Prediction from Histopathological Images of

492 Hepatocellular Carcinoma by a Fully Automated Pipeline Based on Machine Learning. Ann.

493 Surg. Oncol. 27, 2359-2369 (2020).

494 20. Skrede, O.-J. et al. Deep learning for prediction of colorectal cancer outcome: a discovery

495 and validation study. Lancet 395, 350-360 (2020).

496 21. Saillard, C. et al. Predicting survival after hepatocellular carcinoma resection using deep-

497 learning on histological slides. Hepatology (2020). doi:10.1002/hep.31207

498 22. Batts, K. P. \& Ludwig, J. Chronic hepatitis. An update on terminology and reporting.

499 Am. J. Surg. Pathol. 19, 1409-1417 (1995). 
medRxiv preprint doi: https://doi.org/10.1101/2020.08.22.20179952; this version posted August 25, 2020. The copyright holder for this preprint (which was not certified by peer review) is the author/funder, who has granted medRxiv a license to display the preprint in perpetuity.

It is made available under a CC-BY-NC-ND 4.0 International license .

500 23. Sandler, M., Howard, A., Zhu, M., Zhmoginov, A. \& Chen, L.-C. MobileNetV2: Inverted

501 Residuals and Linear Bottlenecks. in 2018 IEEE/CVF Conference on Computer Vision and

502 Pattern Recognition 4510-4520 (IEEE, 2018). doi:10.1109/CVPR.2018.00474

503 24. Russakovsky, O. et al. ImageNet large scale visual recognition challenge. Int J Comput

504 Vis $115,211-252(2015)$.

505 25. Harrell, F. E. Evaluating the yield of medical tests. JAMA 247, 2543 (1982).

506 26. Uno, H., Cai, T., Pencina, M. J., D’Agostino, R. B. \& Wei, L. J. On the C-statistics for

507 evaluating overall adequacy of risk prediction procedures with censored survival data. Stat. Med.

$508 \quad 30,1105-1117(2011)$.

509 27. Mormont, R., Geurts, P. \& Maree, R. Comparison of deep transfer learning strategies for

510 digital pathology. in 2018 IEEE/CVF Conference on Computer Vision and Pattern Recognition

511 Workshops (CVPRW) 2343-234309 (IEEE, 2018). doi:10.1109/CVPRW.2018.00303

512 28. Digestive System Tumours. 635 (International Agency For Research On Cancer Press,

513 IARC, 2019).

514 29. Cancer Genome Atlas Research Network. Electronic address: wheeler@bcm.edu \&

515 Cancer Genome Atlas Research Network. Comprehensive and integrative genomic

516 characterization of hepatocellular carcinoma. Cell 169, 1327-1341.e23 (2017).

517 30. Liu, J. et al. An Integrated TCGA Pan-Cancer Clinical Data Resource to Drive High-

518 Quality Survival Outcome Analytics. Cell 173, 400-416.e11 (2018). 
medRxiv preprint doi: https://doi.org/10.1101/2020.08.22.20179952; this version posted August 25, 2020. The copyright holder for this preprint (which was not certified by peer review) is the author/funder, who has granted medRxiv a license to display the preprint in perpetuity.

It is made available under a CC-BY-NC-ND 4.0 International license .

519 31. Hudis, C. A. et al. Proposal for standardized definitions for efficacy end points in

520 adjuvant breast cancer trials: the STEEP system. J. Clin. Oncol. 25, 2127-2132 (2007).

$521 \quad 32 . \quad$ Vahadane, A. et al. Structure-Preserving Color Normalization and Sparse Stain

522 Separation for Histological Images. IEEE Trans. Med. Imaging 35, 1962-1971 (2016).

523 33. Bilaloglu, S. et al. Efficient pan-cancer whole-slide image classification and outlier

524 detection using convolutional neural networks. BioRxiv (2019). doi:10.1101/633123

525 34. Maas, A. L. Rectifier Nonlinearities Improve Neural Network Acoustic Models. in

526 Proceedings of the 30th International Conference on Machine Learning (2013).

527 35. Glorot, X. \& Bengio, Y. Understanding the difficulty of training deep feedforward neural 528 networks. in Proceedings of the Thirteenth International Conference on Artificial Intelligence

529 and Statistics (eds. Teh, Y. W. \& Titterington, M.) 9, 249-256 (PMLR).

530 36. Kingma, D. P. \& Ba, J. Adam: A Method for Stochastic Optimization. arXiv (2014).

531 37. Loshchilov, I. \& Hutter, F. Decoupled Weight Decay Regularization. arXiv (2017).

532 38. Paszke A, Gross S, Massa F, et al. PyTorch: An Imperative Style, High-Performance

533 Deep Learning Library. https://arxiv.org/abs/1912.01703. Accessed on May 25, 2020.

534 39. Goode A, Gilbert B, Harkes J, Jukic D, Satyanarayanan M. OpenSlide: A Vendor-Neutral 535 Software Foundation for Digital Pathology. J Pathol Inform. 2013Sep 27;4:27. 

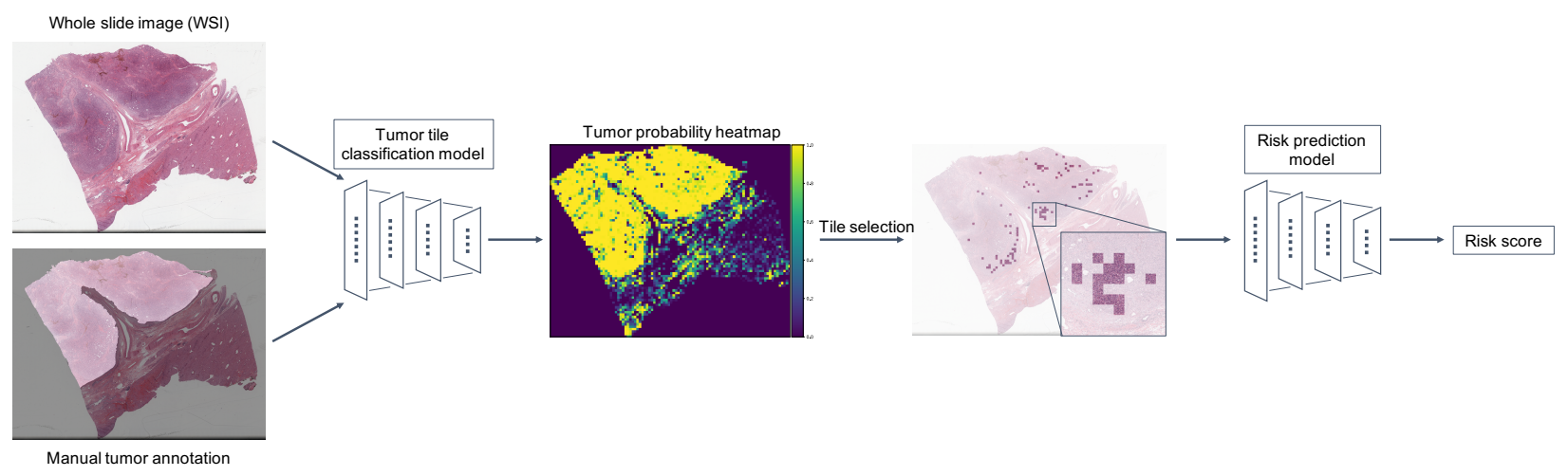

All WSI were preprocessed by discarding non tissue-containing white background using

540 thresholding, then partitioned into non-overlapping tiles of size 299 x 299 pixels and color-

541 normalized. A tumor tile classification model was developed using the Stanford-HCCDET

542 dataset, containing WSI with all tumor regions manually annotated. The tumor tile classification

543 model was subsequently applied to each tissue-containing image tile in the TCGA-HCC $(n=360$

544 WSIs) and Stanford-HCC (n=198 WSIs) datasets for inference. The 100 tiles with the highest

545 predicted probabilities of being tumor tiles were input into the downstream risk prediction model

546 to yield tile-based risk scores, which were averaged to generate a WSI-level risk score for

547 recurrence. Abbreviations: WSI, whole-slide image 
552 Figure 2: Performance of the tumor tile classification model on the internal test set
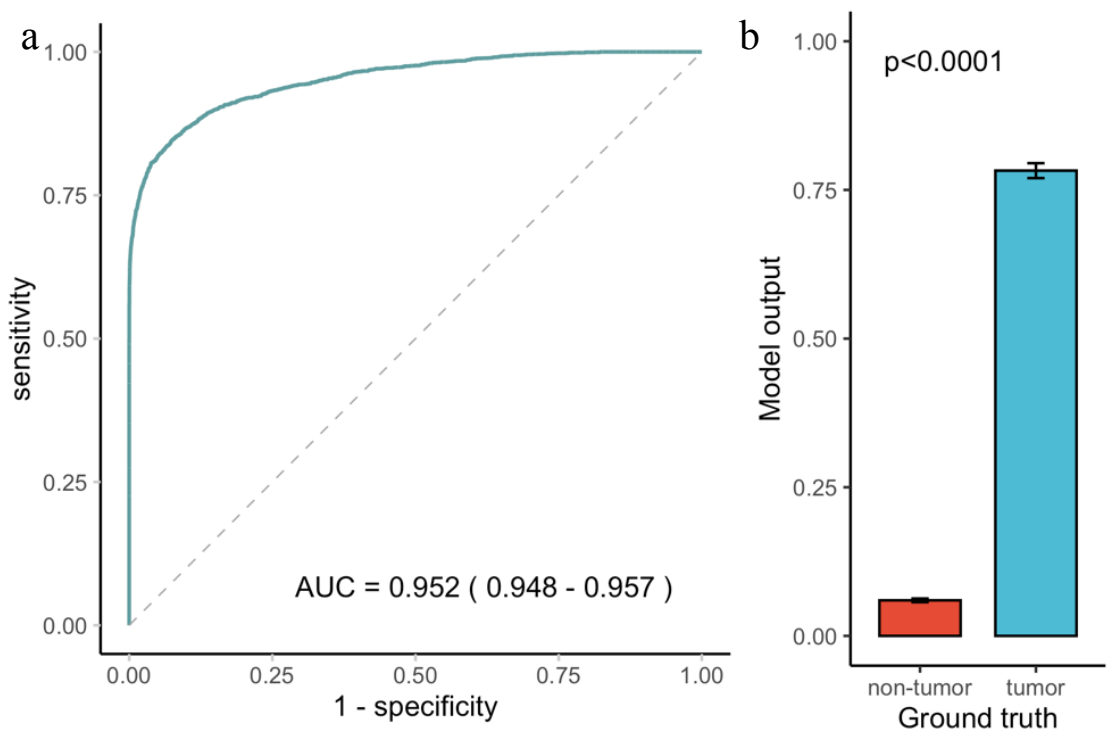

554 The AUROC for tumor tile classification was 0.952 (95\% CI: 0.948, 0.957) on the internal test 555 set (a). Model outputs differed significantly between tiles with a ground truth of tumor versus 556 non-tumor (p-value $<0.0001)$ (b).

$557 *$ The $95 \%$ CI for AUC is shown in parentheses in the ROC plot.

$558 * *$ Error bars represent $95 \% \mathrm{CI}$ in the bar chart. The p-value was computed using the Wilcoxon

559 rank sum test.

560 Abbreviations: AUC, area under the ROC curve; CI, confidence interval; ROC, receiver

561 operating characteristic 
565 Figure 3 Performance of the tumor tile classification model on the external test set
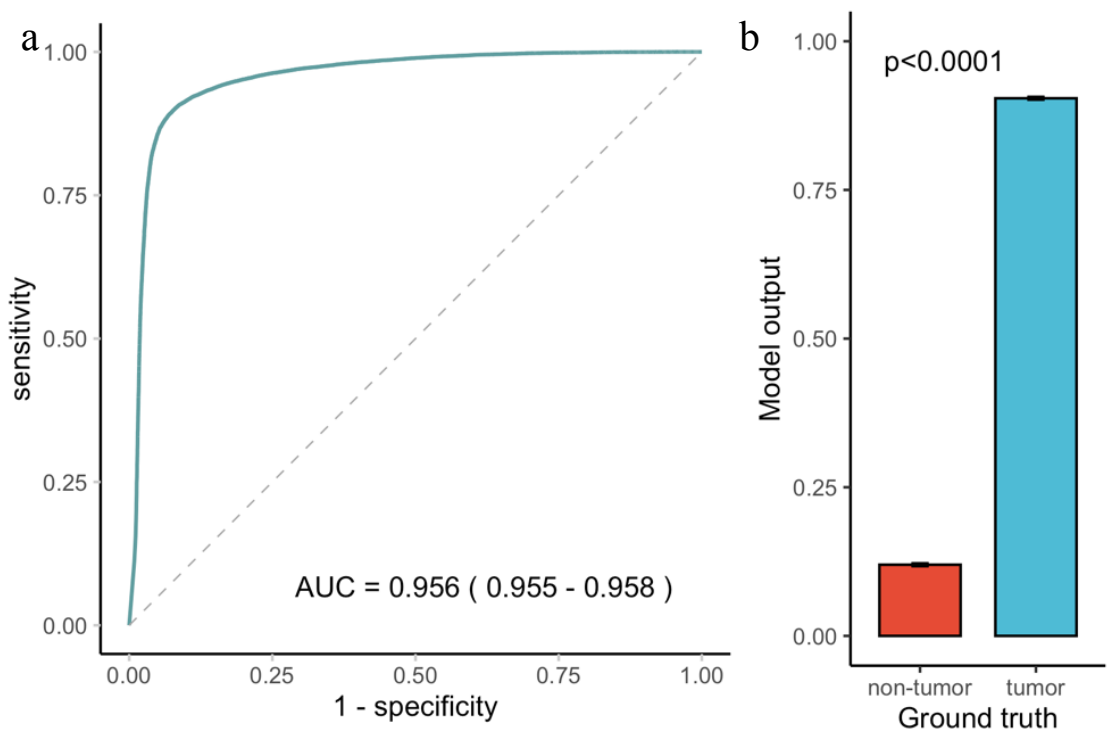

567 The AUROC for tumor tile classification was 0.956 (95\% CI: $0.955,0.958)$ on the external test 568 set (a). Model outputs differed significantly between tiles with a ground truth of tumor versus 569 non-tumor (p-value $<0.0001)$ (b).

$570 * 95 \%$ CI for AUC is shown in parenthesis in the ROC plot.

$571 * *$ Error bars represent $95 \% \mathrm{CI}$ in the bar chart. The p-value was computed using the Wilcoxon 572 rank sum test.

573 Abbreviations: AUC, area under the ROC curve; CI, confidence interval; ROC, receiver

574 operating characteristic

575

576 
578 Figure 4: Top 100 tiles selected by the tumor tile classification model
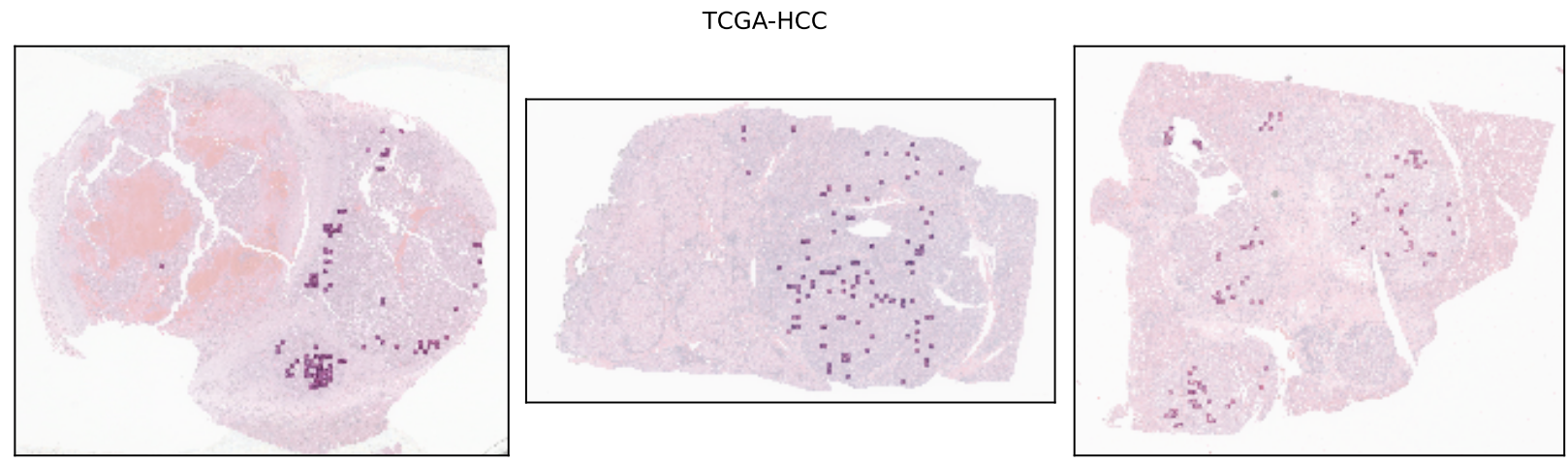

Stanford-HCC
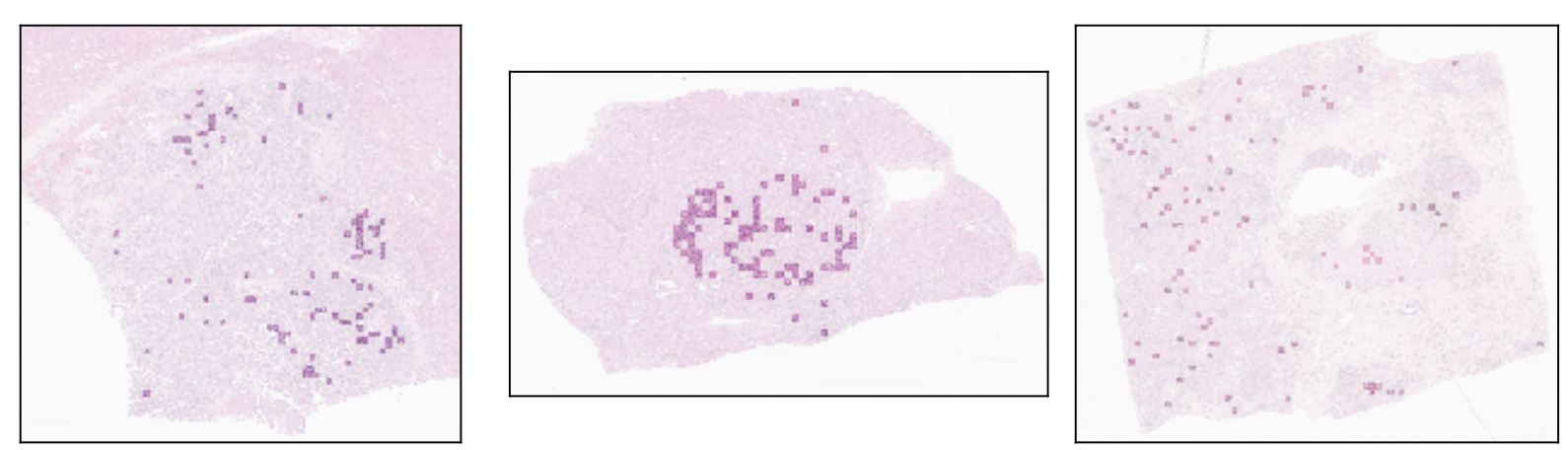

580 Spatial distribution of the top 100 tiles classified as being tumor tiles by the tumor tile

581 classification model. The top row represents examples from the TCGA-HCC test dataset, and the

582 bottom row represents examples from the Stanford-HCC dataset. The top 100 tiles were

583 subsequently used for development of the survival prediction model.

584 Abbreviation: WSI, whole-slide image 
588 Figure 5: Kaplan-Meier plots for the high- and low-risk subgroups in the internal (TCGA-HCC) 589 test set
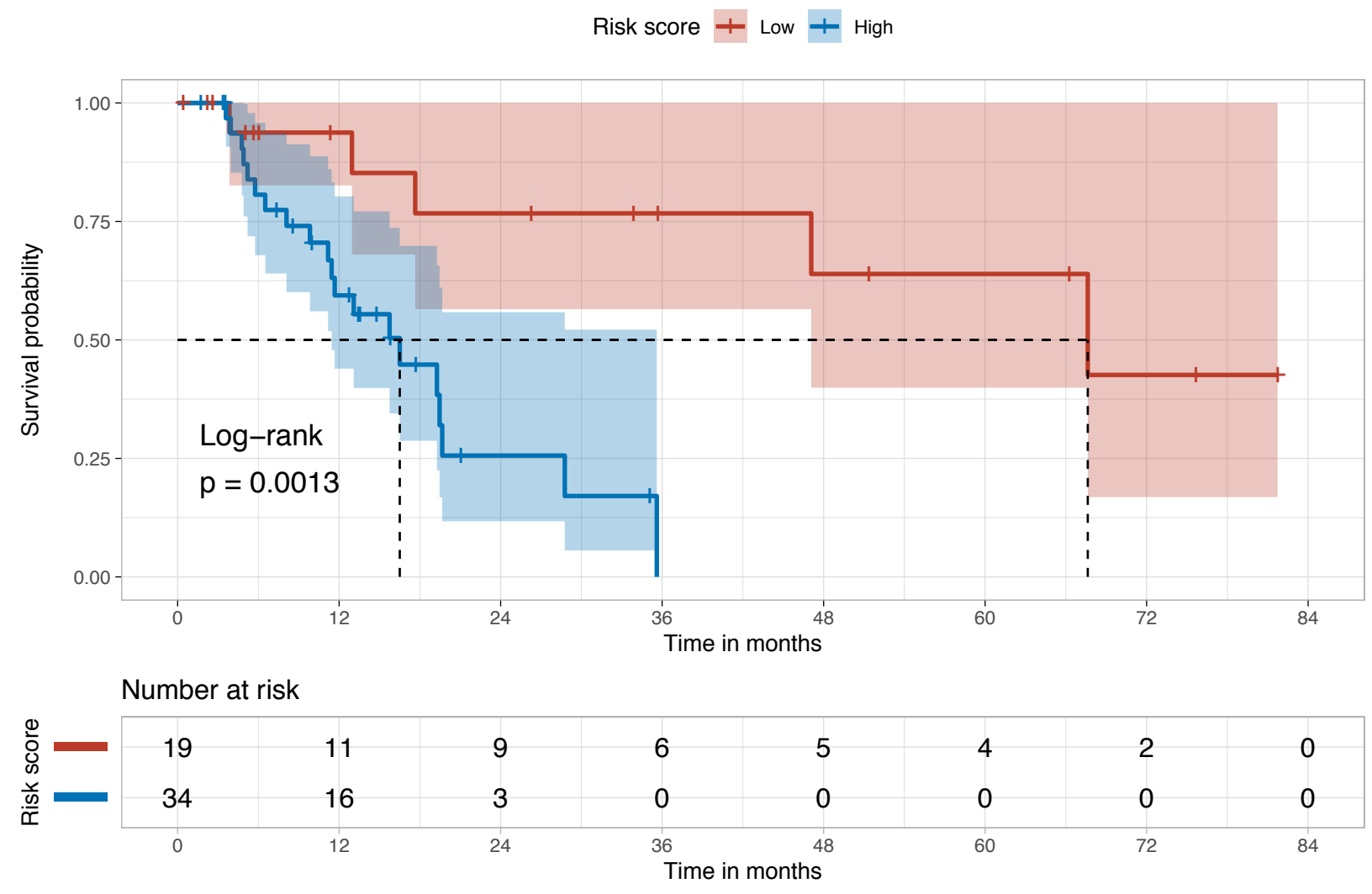

591 The Kaplan-Meier plot shows the difference in the survival distributions for the low- and high-

592 risk subgroups, stratified based on the risk scores predicted by HCC-SurvNet on the internal test

593 set $(\log$-rank p-value $=0.0013)$. 
597 Figure 6: Kaplan-Meier plots for the high- and low-risk subgroups in the external (Stanford598 HCC) test set

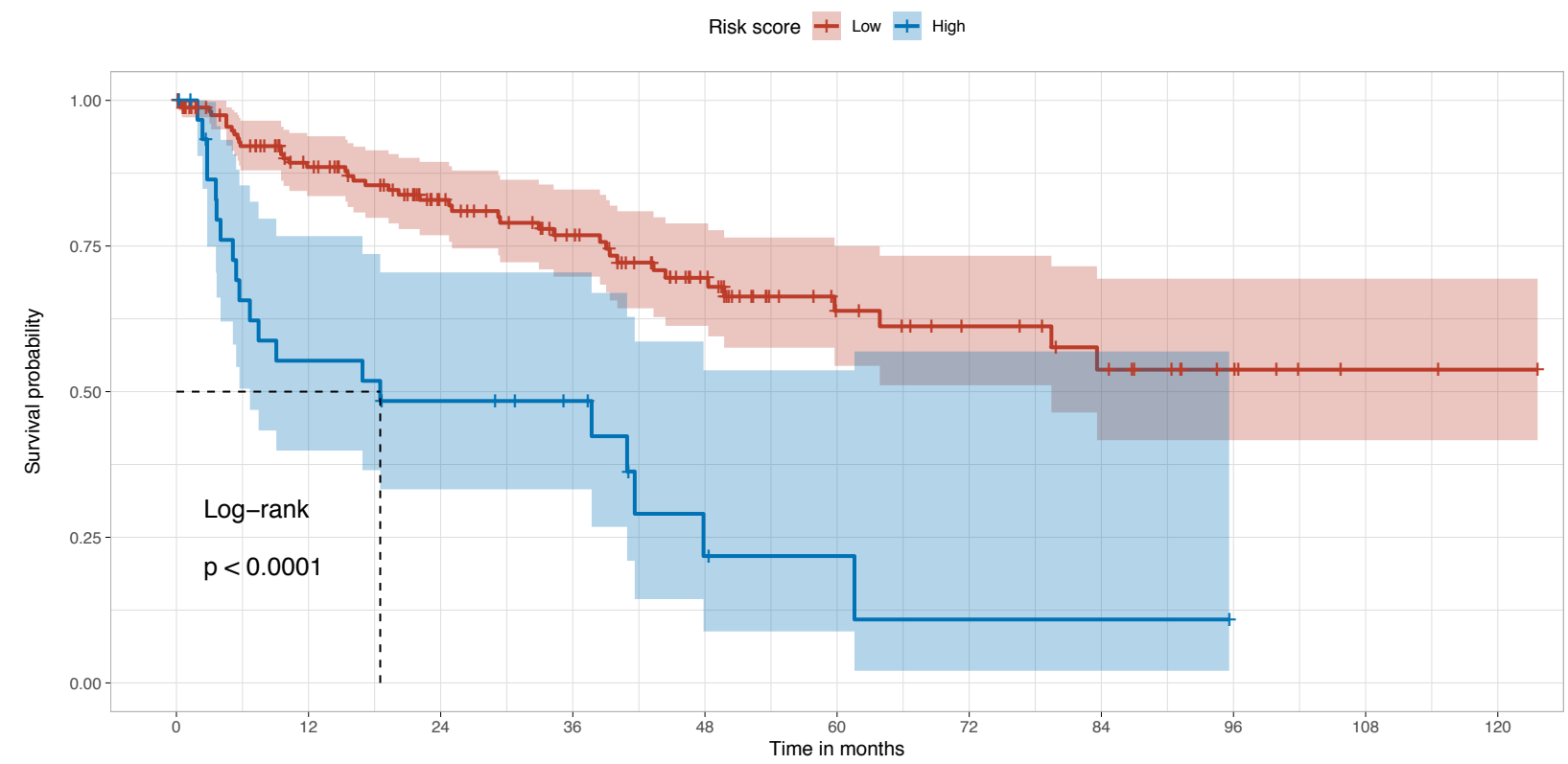

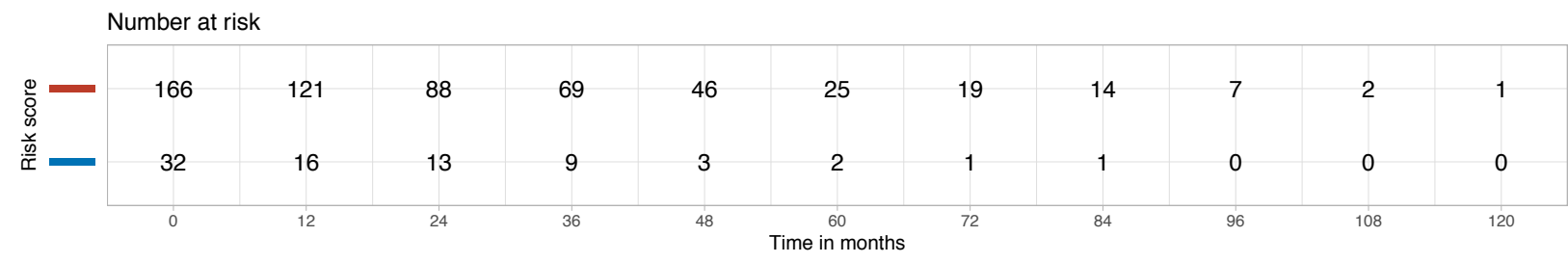

600 The Kaplan-Meier plot shows the difference in the survival distributions for the low- and high-

601 risk subgroups, stratified based on the risk scores predicted by HCC-SurvNet on the external test

602 set $(\log$-rank p-value $<0.0001)$.

604

605 
608 Table 1: Patient characteristics for the Stanford-HCC and TCGA-HCC datasets

\begin{tabular}{cccc}
\hline \multirow{3}{*}{ Patient characteristic } & $\begin{array}{c}\text { TCGA-HCC Development } \\
\text { Cohort }(\mathrm{n}=299)\end{array}$ & $\begin{array}{c}\text { TCGA-HCC Test } \\
\text { Cohort }(\mathrm{n}=53)\end{array}$ & $\begin{array}{c}\text { Stanford-HCC } \\
(\mathrm{n}=198)\end{array}$ \\
& Training and validation set & Internal test set & External test set
\end{tabular}

Age (at surgery) (years)

$60(51,68)$

$61(51,68)$

$64(57,69)$

Gender

Male

$206(69 \%)$

$33(62 \%)$

$157(79 \%)$

Female

$93(31 \%)$

$20(38 \%)$

$41(21 \%)$

Hepatitis B virus infection

Negative

$195(68 \%)$

$33(67 \%)$

$147(74 \%)$

Positive

$90(32 \%)$

$16(33 \%)$

$51(26 \%)$

Unknown

14

4

0

\section{Hepatitis $\mathrm{C}$ virus infection}

Negative

$243(85 \%)$

$41(84 \%)$

$96(48 \%)$

Positive

$42(15 \%)$

$8(16 \%)$

$102(52 \%)$

Unknown

14

4

0

Alcohol intake

Negative

$188(66 \%)$

$30(61 \%)$

$181(91 \%)$

Positive

97 (34\%)

$19(39 \%)$

$17(8.6 \%)$

Unknown

14

4

0

Non-alcoholic fatty liver disease

Negative

$271(95.1 \%)$

$44(90 \%)$

$184(93 \%)$

Positive

$14(4.9 \%)$

$5(10 \%)$

$14(7.1 \%)$

Unknown

14

4

0

\section{AJCC Stage Grouping}

IA

$8(2.7 \%)$

$1(1.9 \%)$

$44(22 \%)$

IB

$121(41 \%)$

$24(46 \%)$

$66(33 \%)$ 
II

IIIA

IIIB

IVA

IVB

Unknown

\section{Largest tumor diameter} (mm)

Unknown

\section{Tumor multifocality}

Negative

Positive

Unknown

\section{Histologic grade}

Well-differentiated

Moderately-

differentiated

Poorly-differentiated

Undifferentiated

\section{Microvascular invasion}

Negative

Positive

Unknown

Macrovascular invasion

Negative

Positive

Unknown

Surgical margin status

Negative

$249(94 \%)$

$17(6.4 \%)$

271 (93\%)

Positive
$85(29 \%)$

$16(31 \%)$

$9(17 \%)$

$1(1.9 \%)$

$0(0 \%)$

$1(1.9 \%)$

1

$65(35,100)$

5

207 (69\%)

$38(73 \%)$

$142(72 \%)$

$91(31 \%)$

$14(27 \%)$

$56(28 \%)$

1

$11(5.6 \%)$

$7(3.5 \%)$

$2(1 \%)$

$0(0 \%)$

0

$30(18,50)$

0

0
$63(32 \%)$

$108(55 \%)$

$26(13 \%)$

$1(0.5 \%)$

$0(0 \%)$

$36(68 \%)$

147 (74\%)

$17(32 \%)$

$51(26 \%)$

0

0

$49(92 \%)$

188 (96\%)

$4(7.5 \%)$

$8(4.1 \%)$

0

0

$45(88 \%)$

$192(97 \%)$ 
Unknown

Fibrosis stage

0
1
2
3
4
Unknown

\section{Recurrence}

No

Yes

Length of follow-up (months)

Risk score
33

$$
\begin{gathered}
77(33 \%) \\
11(4.8 \%) \\
25(11 \%) \\
26(11 \%) \\
91(40 \%) \\
69
\end{gathered}
$$

$148(49 \%)$

$151(51 \%)$

$12(4,24)$
$13(30 \%)$

$2(4.5 \%)$

$4(9.1 \%)$

$6(14 \%)$

$19(43 \%)$

9

$28(53 \%)$

$25(47 \%)$

$13(6,20)$

$0.07(-0.26,0.30)$
$38(19 \%)$

$13(6.6 \%)$

$15(7.6 \%)$

$13(6.6 \%)$

$119(60 \%)$

0

$136(69 \%)$

$62(31 \%)$

$25(9,48)$

$-0.31(-0.46,-0.15)$

609 *Values presented: median (IQR); n (\%)

610

611

612

613

614

615

616

617

618

619

620

621 
622 Table 2: Univariable Cox proportional hazards analysis of the risk of recurrence

\begin{tabular}{|c|c|c|c|c|}
\hline \multirow{3}{*}{ Patient characteristics } & \multicolumn{2}{|c|}{$\begin{array}{l}\text { TCGA-HCC Test Cohort } \\
\qquad(\mathrm{n}=53)\end{array}$} & \multicolumn{2}{|c|}{ Stanford-HCC $(\mathrm{n}=198)$} \\
\hline & \multicolumn{2}{|c|}{ Internal test set } & \multicolumn{2}{|c|}{ External test set } \\
\hline & $\begin{array}{l}\text { Hazard ratio }(95 \% \\
\text { CI) }\end{array}$ & $\mathrm{p}$ value & $\begin{array}{l}\text { Hazard ratio }(95 \% \\
\text { CI) }\end{array}$ & $\mathrm{p}$ value \\
\hline Risk score (binarized) & $6.52(1.83,23.2)$ & 0.0038 & $3.72(2.17,6.37)$ & $<0.0001$ \\
\hline \multicolumn{5}{|l|}{ Age (at surgery) } \\
\hline$>60$ & $0.83(0.38,1.8)$ & 0.65 & $1.1(0.64,1.8)$ & 0.77 \\
\hline \multicolumn{5}{|l|}{ Gender } \\
\hline Female & $0.99(0.44,2.2)$ & 0.98 & $0.99(0.53,1.9)$ & 0.98 \\
\hline \multicolumn{5}{|l|}{ Hepatitis B virus infection } \\
\hline Positive & $0.57(0.22,1.5)$ & 0.25 & $1.1(0.62,1.9)$ & 0.78 \\
\hline \multicolumn{5}{|l|}{ Hepatitis $C$ virus infection } \\
\hline Positive & $1.2(0.4,3.6)$ & 0.74 & $0.66(0.4,1.1)$ & 0.11 \\
\hline \multicolumn{5}{|l|}{ Alcohol intake } \\
\hline Positive & $0.89(0.37,2.2)$ & 0.80 & $0.19(0.027,1.4)$ & 0.10 \\
\hline \multicolumn{5}{|l|}{ Non-alcoholic fatty liver disease } \\
\hline Positive & $1.6(0.46,5.4)$ & 0.48 & $1.8(0.81,3.9)$ & 0.15 \\
\hline \multicolumn{5}{|l|}{ AJCC Stage grouping } \\
\hline$>$ II & $1.3(0.49,3.6)$ & 0.58 & $4.4(2.3,8.3)$ & $<0.0001$ \\
\hline \multicolumn{5}{|l|}{ Largest tumor diameter $(\mathbf{m m})$} \\
\hline$>50$ & $1.1(0.52,2.5)$ & 0.74 & $3.5(2.1,5.8)$ & $<0.0001$ \\
\hline \multicolumn{5}{|l|}{ Tumor multifocality } \\
\hline Positive & $1.3(0.53,3.4)$ & 0.53 & $1.1(0.64,1.9)$ & 0.71 \\
\hline \multicolumn{5}{|l|}{ Histologic grade } \\
\hline$>$ Moderately-differentiated & $1.1(0.44,2.6)$ & 0.90 & $2.1(1.2,3.9)$ & 0.013 \\
\hline \multicolumn{5}{|l|}{ Microvascular invasion } \\
\hline Positive & $1.4(0.6,3.1)$ & 0.46 & $3.9(2.4,6.5)$ & $<0.0001$ \\
\hline
\end{tabular}


$\begin{array}{lllll}\text { Positive } & 1.6(0.46,5.4) & 0.48 & 5.3(2.1,1.3) & 0.00043\end{array}$

Surgical margin

$\begin{array}{lllll}\text { Positive } & 0.74(0.22,2.5) & 0.63 & 6.8(1.6,28) & 0.0090\end{array}$

Fibrosis stage

$\begin{array}{lllll}>2 & 2.7(0.98,7.7) & 0.054 & 0.33(0.2,0.55) & <0.0001\end{array}$

$623 *$ CI denotes confidence interval.

624

625 Table 3: Multivariable Cox proportional hazards analysis of the risk of recurrence

\begin{tabular}{|c|c|c|c|c|}
\hline \multirow{3}{*}{ Patient characteristics } & \multirow{2}{*}{\multicolumn{2}{|c|}{$\begin{array}{l}\text { TCGA-HCC Test Cohort } \\
\qquad(\mathrm{n}=53) \\
\text { Internal test set }\end{array}$}} & \multicolumn{2}{|c|}{ Stanford-HCC $(n=198)$} \\
\hline & & & \multicolumn{2}{|c|}{ External test set } \\
\hline & $\begin{array}{c}\text { Hazard ratio }(95 \% \\
\mathrm{CI})\end{array}$ & $\mathrm{p}$ value & $\begin{array}{l}\text { Hazard ratio }(95 \% \\
\text { CI })\end{array}$ & $\mathrm{p}$ value \\
\hline Risk score (binarized) & $7.44(1.60,34.6)$ & 0.011 & $2.37(1.27,4.43)$ & 0.00685 \\
\hline \multicolumn{5}{|l|}{ AJCC Stage grouping } \\
\hline$>$ II & $0.30(0.043,2.11)$ & 0.23 & $1.57(0.63,3.91)$ & 0.331 \\
\hline \multicolumn{5}{|l|}{ Largest tumor diameter $(\mathrm{mm})$} \\
\hline$>50$ & $1.09(0.33,3.60)$ & 0.89 & $1.32(0.67,2.60)$ & 0.425 \\
\hline \multicolumn{5}{|l|}{ Histologic grade } \\
\hline$>$ Moderately-differentiated & $2.82(0.98,8.1)$ & 0.054 & $1.36(0.69,2.69)$ & 0.377 \\
\hline \multicolumn{5}{|l|}{ Microvascular invasion } \\
\hline Positive & $1.98(0.59,6.64)$ & 0.27 & $2.84(1.61,5.00)$ & 0.000294 \\
\hline \multicolumn{5}{|l|}{ Macrovascular invasion } \\
\hline Positive & $3.76(0.77,18.4)$ & 0.10 & $1.42(0.42,4.86)$ & 0.575 \\
\hline \multicolumn{5}{|l|}{ Surgical margin } \\
\hline Positive & $0.51(0.079,3.3)$ & 0.47 & $4.45(0.84,23.7)$ & 0.0797 \\
\hline \multicolumn{5}{|l|}{ Fibrosis stage } \\
\hline$>2$ & $0.80(0.20,3.1)$ & 0.74 & $0.50(0.28,0.90)$ & 0.0217 \\
\hline
\end{tabular}

$626 *$ CI denotes confidence interval. 
627 Table 4: Association between the HCC-SurvNet risk score and various patient characteristics in 628 the external test (Stanford-HCC) cohort

\begin{tabular}{|c|c|c|c|c|}
\hline \multirow[b]{2}{*}{ Patient characteristics } & \multicolumn{2}{|c|}{ Stanford-HCC $(n=198)$} & \multicolumn{2}{|c|}{ Spearman's correlation } \\
\hline & $\begin{array}{c}\text { Low-risk } \\
\text { group }(\mathrm{N}= \\
166)\end{array}$ & $\begin{array}{c}\text { High-risk } \\
\text { group }(\mathrm{N}=32)\end{array}$ & $\rho(95 \% \mathrm{CI})$ & $\mathrm{p}$ value \\
\hline Age (at surgery) & $64(57,69)$ & $64(57,69)$ & $-0.017(-0.15,0.12)$ & 0.82 \\
\hline Gender & & & $0.036(-0.11,0.18)$ & 0.62 \\
\hline Male & $133(80 \%)$ & $24(75 \%)$ & & \\
\hline Female & $33(20 \%)$ & $8(25 \%)$ & & \\
\hline Hepatitis B virus infection & & & $-0.033(-0.17,0.10)$ & 0.64 \\
\hline Negative & $121(73 \%)$ & $26(81 \%)$ & & \\
\hline Positive & $45(27 \%)$ & $6(19 \%)$ & & \\
\hline Hepatitis $C$ virus infection & & & $-0.082(-0.22,0.063)$ & 0.25 \\
\hline Negative & $80(48 \%)$ & $16(50 \%)$ & & \\
\hline Positive & $86(52 \%)$ & $16(50 \%)$ & & \\
\hline Alcohol intake & & & $-0.024(-0.18,0.13)$ & 0.74 \\
\hline Negative & $152(92 \%)$ & $29(91 \%)$ & & \\
\hline Positive & $14(8.4 \%)$ & $3(9.4 \%)$ & & \\
\hline $\begin{array}{l}\text { Non-alcoholic fatty liver } \\
\text { disease }\end{array}$ & & & $-0.0014(-0.10,0.092)$ & 0.99 \\
\hline Negative & $152(92 \%)$ & $32(100 \%)$ & & \\
\hline Positive & $14(8.4 \%)$ & $0(0 \%)$ & & \\
\hline AJCC Stage grouping & & & $0.24(0.086,0.37)$ & 0.00079 \\
\hline IA & $43(26 \%)$ & $1(3.1 \%)$ & & \\
\hline IB & $53(32 \%)$ & $13(41 \%)$ & & \\
\hline II & $57(34 \%)$ & $11(34 \%)$ & & \\
\hline IIIA & $7(4.2 \%)$ & $4(12 \%)$ & & \\
\hline IIIB & $5(3.0 \%)$ & $2(6.2 \%)$ & & \\
\hline IVA & $1(0.6 \%)$ & $1(3.1 \%)$ & & \\
\hline
\end{tabular}


IVB

Largest tumor diameter (mm)

Tumor multifocality

Negative

Positive

Histologic grade

Well-differentiated

Moderately-differentiated

Poorly-differentiated

Undifferentiated

Microvascular invasion

Negative

Positive

Macrovascular invasion

Negative

Positive

\section{Surgical margin status}

Negative

Positive

Unknown

Fibrosis stage

0

1

2

3

4

$$
0(0 \%)
$$

$26(15,45)$

$188(71 \%)$

$48(29 \%)$

57 (34\%)

89 (54\%)

$19(11 \%)$

$1(0.6 \%)$

$128(77 \%)$

$38(23 \%)$

$159(97 \%)$

$5(3.0 \%)$

159 (97\%)

$5(3.0 \%)$

2

$29(17 \%)$

$8(4.8 \%)$

$12(7.2 \%)$

$9(5.4 \%)$

$108(65 \%)$
$0(0 \%)$

$55(36,80)$

$0.41(0.27,0.53)$

$<0.0001$

$-0.10(-0.24,0.032)$

0.15

$24(75 \%)$

$8(25 \%)$

$0.14(-0.0046,0.27)$

0.058

$6(19 \%)$

$19(59 \%)$

$7(22 \%)$

$0(0 \%)$

$0.22(0.082,0.35)$

0.0015

$19(59 \%)$

$13(41 \%)$

$0.056(-0.13,0.23)$

0.44

$29(91 \%)$

$3(9.4 \%)$

$0.060(-0.079,0.18)$

0.40

$29(91 \%)$

$3(9.4 \%)$

0

$629 *$ Values presented: median (IQR); n (\%)

630 


\section{Supplementary Figures}

Supplementary Figure 1: Histograms of HCC-SurvNet Risk Scores

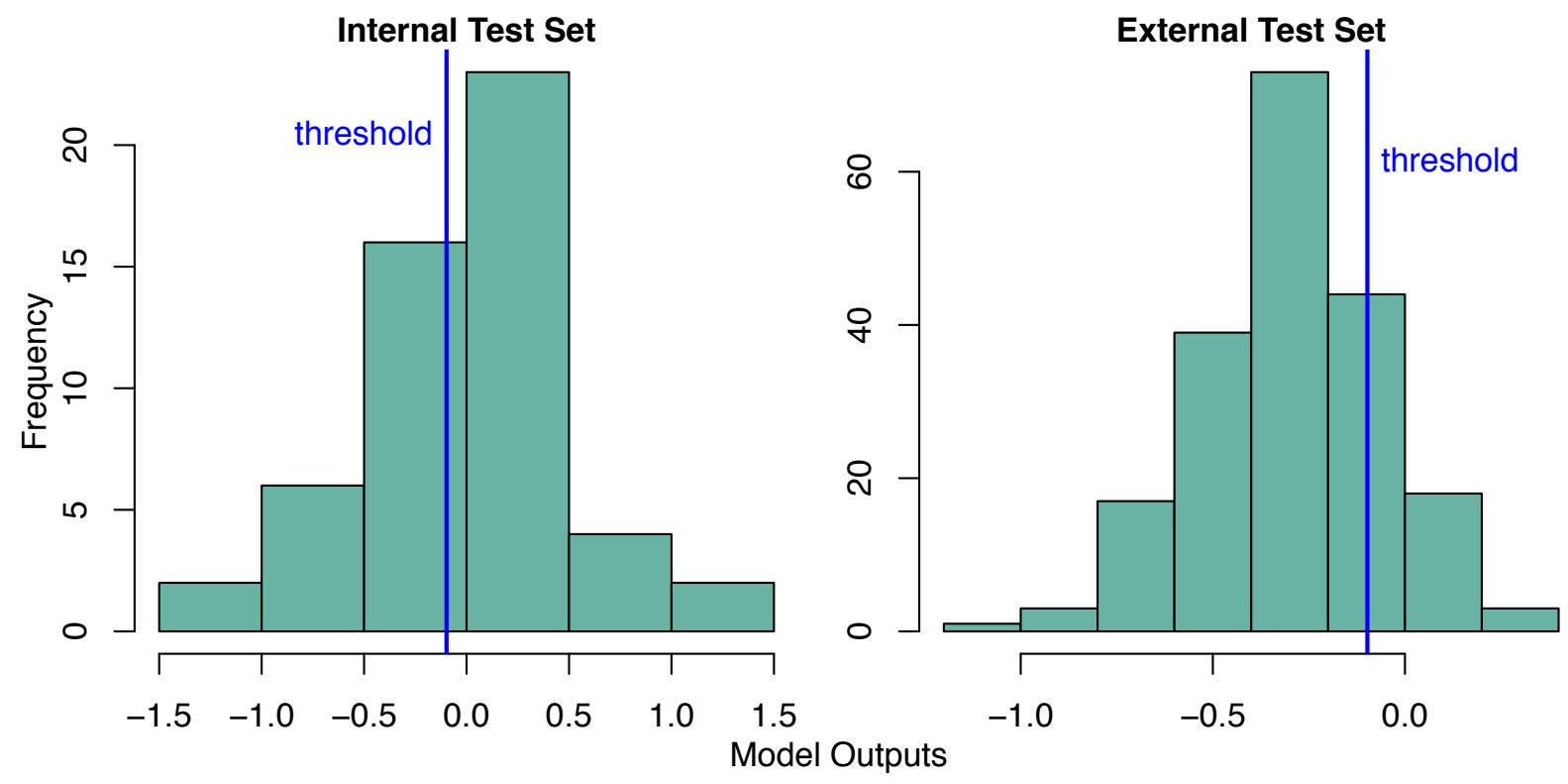

The histograms show the distributions of HCC-SurvNet's risk scores within the internal (left) and external (external) test sets separately. A threshold used for patient stratification into lowand high-risk groups, which was determined on the validation set from TCGA-HCC, is visualized as a blue vertical line (threshold $=-0.0978)$. 
Supplementary Figure 2: Univariable Cox Proportional Hazards Regression Analysis with Restricted Cubic Splines
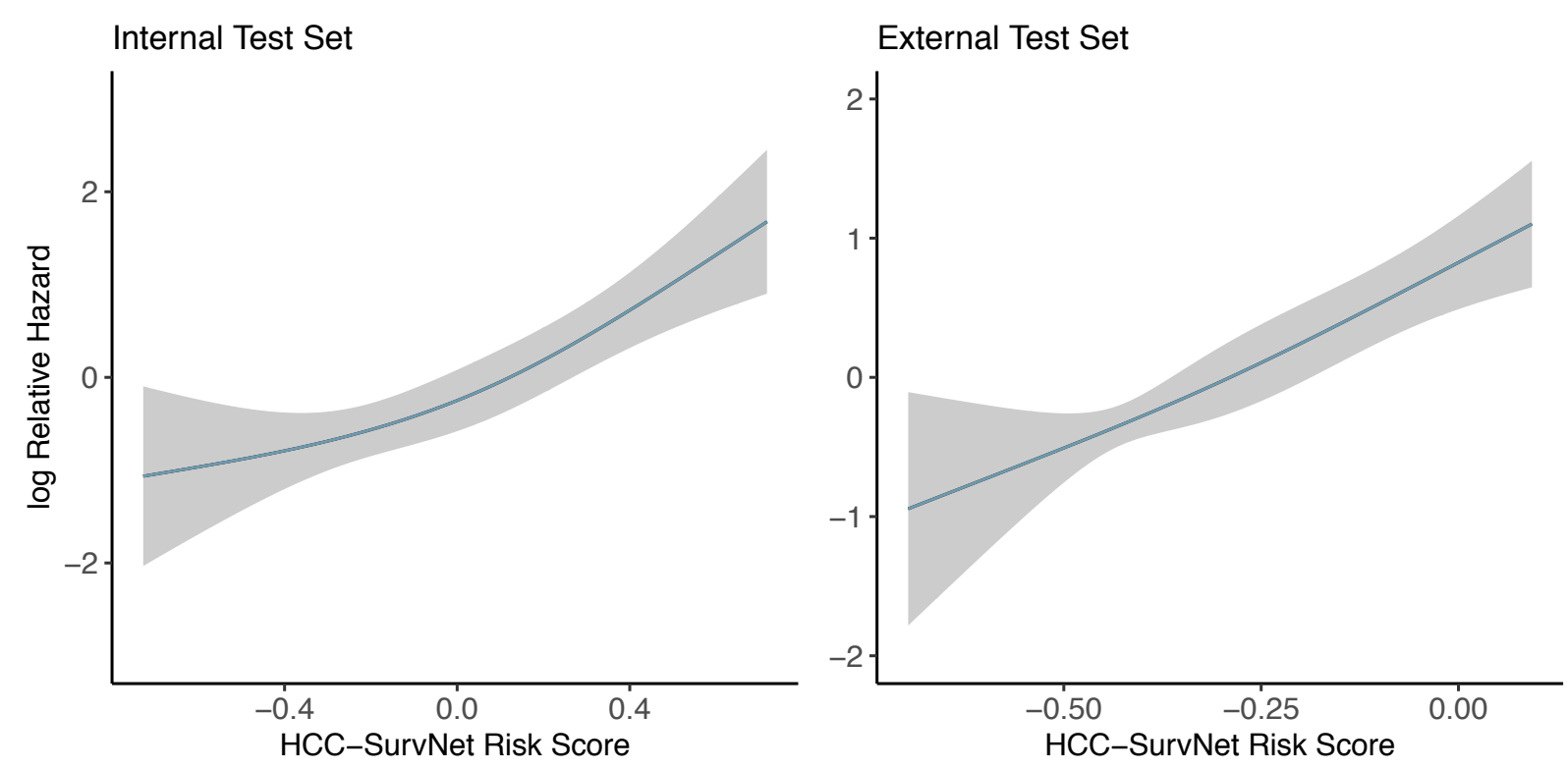

A continuous linear association between HCC-SurvNet's risk score and the log relative hazard for RFI was observed upon analysis of the internal test set (left), and even more significantly for the external test set (right). The blue line represents the fitted line of the association between HCC-SurvNet's risk score and the log relative hazard for RFI; the shaded region represents the $95 \%$ CI.

Abbreviations: CI, confidence interval; RFI, recurrence-free interval 